Article

\title{
Chemical Constituents of the Root of Jasminum giraldii
}

Zhenggang Yue ${ }^{1,2, \dagger}$, Hui Qin ${ }^{3, \dagger}$, Yuhua Li $^{1,2}$, Yang Sun ${ }^{1,2}$, Zhipeng Wang ${ }^{1,2}$, Tiehong Yang ${ }^{1,2}$, Li Liu ${ }^{1,2}$, Minchang Wang ${ }^{4}$, Feng Feng ${ }^{3, *}$ and Qibing Mei ${ }^{1,2, *}$

1 Department of Pharmacology, School of Pharmacy, the Fourth Military Medical University, Xi'an 710032, Shaanxi, China

2 Collaborative Innovation Center for Chinese Medicine in Qingba Mountains, the Fourth Military Medical University, Xi'an 710032, Shaanxi, China

3 Department of Natural Medicinal Chemistry, China Pharmaceutical University, Nanjing 210009, Jiangsu, China

4 Xi'an Modern Chemistry Research Institute, Xi'an 710032, Shaanxi, China

$\dagger$ These authors contributed equally to this work.

* Authors to whom correspondence should be addressed; E-Mails: fengfeng@cpu.edu.cn (F.F.), qbmei@fmmu.edu.cn (Q.M.); Tel.: +86-139-5204-5795 (F.F.); Fax: +86-25-8327-1269 (F.F.); Tel./Fax: +86-29-8477-9212 (Q.M.).

Received: 1 February 2013; in revised form: 26 March 2013 / Accepted: 12 April 2013 / Published: 22 April 2013

Abstract: Two new compounds, ethylconiferin (1) and (-)-lariciresinol 4-(6"'-O-cinnamyl- $\beta$-D-glucopyranoside) (2), along with the three known compounds $(+)$-pinoresinol (3), (+)-cycloolivil (4), nobiletin (5), were isolated from the root of Jasminum girialdii. All these compounds were isolated for the first time from this source. Their structures were elucidated on the basis of extensive spectroscopic analysis and chemical methods. In addition, the in vitro cytotoxic activity of these compounds was evaluated. The results showed that none of the compounds had any significant inhibitory activities on the proliferation of HCT-116 and SW-620 cells.

Keywords: Jasminum giraldii; oleaceae; chemical constituents; cytotoxic activity 


\section{Introduction}

Jasminum giraldii Diels (Oleaceae), is an endemic plant which is found distributed at an altitude of 300-1,500 m, in valleys and shrubbery of the Qinba Mountains in China's Shaanxi Province [1], Its dried roots, named as "quan pi" in Chinese, are used as a traditional local herb for the treatment of various diseases, such as fractures, traumatic injury and blood stasis, while some other species of this genus are employed to treat dysmenorrhea, metritis, leucorrhoea, galactophoritis, puerperal infection, irregular menstruation, hyperthermia, arthralgia, pain due to ischemia, dermatitis [2], fever, rheumatic pain [3], dysenteric diarrhea, trachelopanus, eczema [4], giddiness, edema [5] in Traditional Chinese Medicine.

The chemistry and pharmacology of the ethanol extracts of the roots of $J$. giraldii have never been systematically investigated. As part of a program to acquire and assess potent chemicals in several traditional Chinese medicines for the preservation and treatment of colorectal cancer [6-9], an ethanolic extract of the root of $J$. giraldii has been investigated. We describe herein the isolation and structural elucidation of two new compounds 1,2 (Figure 1) along with three known compounds 3-5 [10-12]. Furthermore, the cytotoxic activity of these compounds against HCT-116 and SW-620 cells is reported for the first time.

Figure 1. The structures of compounds $\mathbf{1}-\mathbf{5}$.

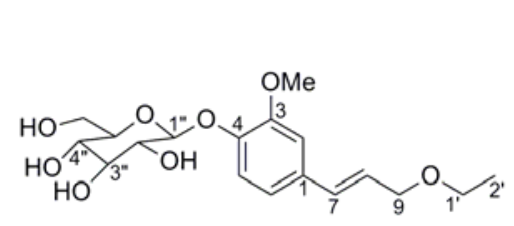

1

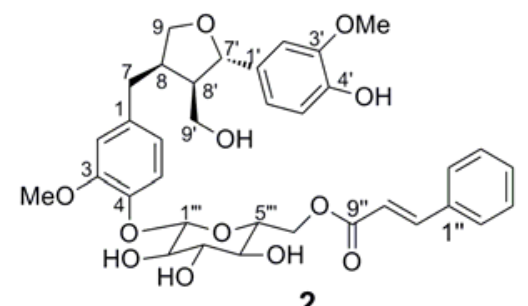

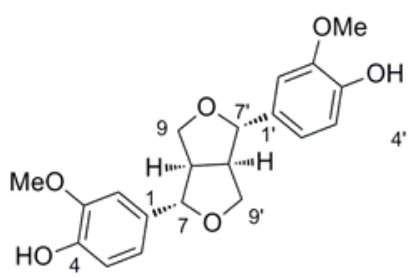

3

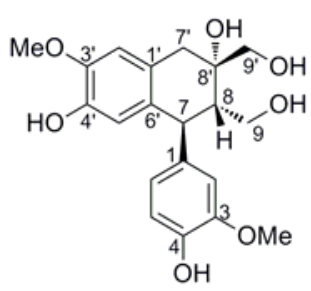

4

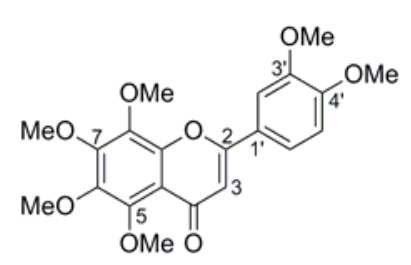

5

\section{Results and Discussion}

Compound 1 was obtained as a white powder, whose molecular formula $\mathrm{C}_{18} \mathrm{H}_{26} \mathrm{O}_{8}$ was indicated by HR-TOF-MS at $m / z 393.1525[\mathrm{M}+\mathrm{Na}]^{+}$(calcd for $\mathrm{C}_{18} \mathrm{H}_{26} \mathrm{O}_{8} \mathrm{Na}, 393.1525$ ), implying six degrees of unsaturation. The max absorptions in the UV spectrum of 1 were observed at 290, 259, $213 \mathrm{~nm}$ (in $\left.\mathrm{CH}_{3} \mathrm{OH}\right)$. The IR spectrum of 1 showed absorption bands of hydroxyl $\left(3496,3444,3367 \mathrm{~cm}^{-1}\right)$, aromatic ring $\left(1510,1454 \mathrm{~cm}^{-1}\right)$, and olefinic $\left(1649 \mathrm{~cm}^{-1}\right)$ functionalities. The ${ }^{1} \mathrm{H}-\mathrm{NMR}$ spectra of 1 (Table 1) in $\mathrm{CD}_{3} \mathrm{OD}$ showed signals attributed to an aromatic ring $\mathrm{ABX}$ coupled system at $\ddot{a} 7.15(1 \mathrm{H}$, $\mathrm{d}, J=8.35 \mathrm{~Hz}, \mathrm{H}-5), 7.11(1 \mathrm{H}, \mathrm{d}, J=1.90 \mathrm{~Hz}, \mathrm{H}-2)$ and $6.99(1 \mathrm{H}, \mathrm{dd}, J=1.90,8.35 \mathrm{~Hz}, \mathrm{H}-6)$, together with signals attributed to an aromatic methoxy group at $\ddot{a} 3.90(3 \mathrm{H}, \mathrm{s})$, The presence of a trans-arylpropenoxy unit was characterized by signals at $\ddot{a} 6.61(1 \mathrm{H}, \mathrm{d}, J=15.9 \mathrm{~Hz}, \mathrm{H}-7), 6.26(1 \mathrm{H}$, dt, $J=6.15,15.9 \mathrm{~Hz}, \mathrm{H}-8)$ and $4.15(2 \mathrm{H}, \mathrm{dd}, J=1.25,6.15 \mathrm{~Hz}, \mathrm{H}-9)$. Meanwhile, an ethoxyl unit was 
indicated by signals of $3.60\left(2 \mathrm{H}, \mathrm{q}, J=7.05 \mathrm{~Hz}, \mathrm{H}-1^{\prime}\right), 1.25\left(3 \mathrm{H}, \mathrm{t}, J=7.05 \mathrm{~Hz}, \mathrm{H}-2^{\prime}\right)$, In addition, a doublet assignable to an anomeric proton at $\delta 4.93(1 \mathrm{H}, \mathrm{d}, J=7.25 \mathrm{~Hz}, \mathrm{H}-1 ")$, together with partially overlapped signals attributed to oxymethylene and oxymethine protons at $\delta 3.90(1 \mathrm{H}, \mathrm{dd}, \mathrm{H}-6 \mathrm{a} "), 3.72$ (1H, dd, $J=7.45,12.0$ Hz, H-6b"), 3.52 (1H, m, H-2"), 3.51 (1H, m, H-3"), 3.50 (1H, m, H-5"), 3.48 $(1 \mathrm{H}, \mathrm{m}, \mathrm{H}-4 ")$, suggested that there was a glycosyl moiety with a $\beta$ configuration in $\mathbf{1}$. Enzymatic hydrolysis of 1 produced a sugar, which was identified as glucose by its ${ }^{1} \mathrm{H}-\mathrm{NMR}$ data and TLC comparison with an authentic sugar sample. The glucose isolated from the hydrolysate gave a positive optical rotation $[\alpha]_{D}^{20}:+47.2$, indicating that it was D-glucose [13]. The ${ }^{13} \mathrm{C}-\mathrm{NMR}$ and DEPT spectra of 1 showed carbon signals corresponding to the above units (Table 1). The HMBC correlation signals (Table 1 and Figure 2$)$ of $\mathrm{H}-1 "\left(\delta_{\mathrm{H}} 4.93\right) / \mathrm{C}-4\left(\delta_{\mathrm{C}} 147.96\right)$ indicated that the glucosyl group was linked to $\mathrm{C}-4, \mathrm{H}-7 \quad\left(\delta_{\mathrm{H}} 6.61\right) / \mathrm{C}-1 \quad\left(\delta_{\mathrm{C}} 133.38\right), \mathrm{C}-2 \quad\left(\delta_{\mathrm{C}} 111.59\right), \mathrm{C}-6 \quad\left(\delta_{\mathrm{C}} 121.0\right)$ indicated that the trans-arylpropenoxy unit was connected to the aromatic ring via a $\mathrm{C}-7-\mathrm{C}-1$ bond, $\mathrm{H}-1$ ' $\left(\delta_{\mathrm{H}} 3.60\right) / \mathrm{C}-9$ $\left(\delta_{\mathrm{C}} 72.38\right)$ indicated that the ethoxyl unit was linked to C-9. Therefore, compound $\mathbf{1}$, whose ${ }^{1} \mathrm{H}-\mathrm{NMR}$ and ${ }^{13} \mathrm{C}-\mathrm{NMR}$ were similar to those of methylconiferin [14], was named ethylconiferin.

Figure 2. Key HMBC correlations in compound 1.

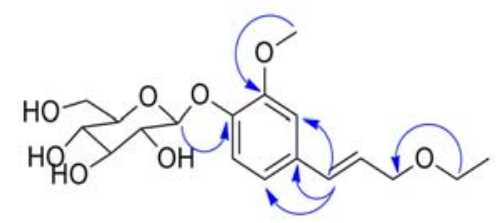

Table 1. ${ }^{1} \mathrm{H}-\mathrm{NMR}(500 \mathrm{MHz})$ and ${ }^{13} \mathrm{C}-\mathrm{NMR}(125 \mathrm{MHz})$ spectral data and HMBC Correlations of Compound $\mathbf{1}$ in $\mathrm{CD}_{3} \mathrm{OD}$.

\begin{tabular}{cccc}
\hline NO. & $\boldsymbol{\delta}_{\mathbf{C}}$ & $\boldsymbol{\delta}_{\mathbf{H}}(\boldsymbol{J}, \mathbf{H z})$ & HMBC \\
\hline $\mathbf{1}$ & 133.38 & & \\
$\mathbf{2}$ & 111.59 & $7.11(1 \mathrm{H}, \mathrm{d}, 1.9)$ & 4,6 \\
$\mathbf{3}$ & 151.07 & & \\
$\mathbf{4}$ & 147.96 & & 1,3 \\
$\mathbf{5}$ & 118.08 & $7.15(1 \mathrm{H}, \mathrm{d}, 8.35)$ & $1,2,4,5$ \\
$\mathbf{6}$ & 121.0 & $6.99(1 \mathrm{H}, \mathrm{dd}, 1.9,8.35)$ & $1,2,6,9$ \\
$\mathbf{7}$ & 133.55 & $6.61(1 \mathrm{H}, \mathrm{d}, 15.9)$ & 1,9 \\
$\mathbf{8}$ & 126.13 & $6.26(1 \mathrm{H}, \mathrm{dt}, 6.15,15.85)$ & $1,1^{\prime}, 8$ \\
$\mathbf{9}$ & 72.38 & $4.15(2 \mathrm{H}, \mathrm{dd}, 1.25,6.15)$ & $2^{\prime}, 9$ \\
$\mathbf{1}$ & 66.78 & $3.60(2 \mathrm{H}, \mathrm{q}, 7.05)$ & $1^{\prime}$ \\
$\mathbf{2}^{\prime}$ & 15.59 & $1.25(3 \mathrm{H}, \mathrm{t}, 7.05)$ & $1^{\prime \prime}, 3^{\prime \prime}$ \\
$\mathbf{1 "}$ & 102.89 & $4.93(1 \mathrm{H}, \mathrm{d}, 7.25)$ & $2^{\prime \prime}, 4^{\prime \prime}$ \\
$\mathbf{2}^{\prime \prime}$ & 75.05 & $3.52(1 \mathrm{H}, \mathrm{m})$ & $5^{\prime \prime}$ \\
$\mathbf{3}^{\prime \prime}$ & 78.00 & $3.51(1 \mathrm{H}, \mathrm{m})$ & \\
$\mathbf{4}^{\prime \prime}$ & 71.49 & $3.48(1 \mathrm{H}, \mathrm{m})$ & \\
$\mathbf{5}^{\prime \prime}$ & 78.38 & $3.50(1 \mathrm{H}, \mathrm{m})$ & 3 \\
$\mathbf{6 a}^{\prime}$ & 62.66 & $3.90(1 \mathrm{H}, \mathrm{dd})$ & \\
$\mathbf{6 b}^{\prime \prime}$ & & $3.72(1 \mathrm{H}, \mathrm{dd}, 7.45,12)$ & \\
$\mathbf{3 - O C H _ { 3 }}$ & 56.87 & $3.90(3 \mathrm{H}, \mathrm{s})$ & \\
\hline
\end{tabular}


Compound 2 was isolated as a white powder, whose molecular formula $\mathrm{C}_{35} \mathrm{H}_{40} \mathrm{O}_{12}$ was determined by HR-TOF-MS at $m / z 653.2589[\mathrm{M}+\mathrm{H}]^{+}$(calcd for $\mathrm{C}_{35} \mathrm{H}_{41} \mathrm{O}_{12}, 652.2590$ ), The UV absorption bands of 2 appeared at 278, 216 and $203 \mathrm{~nm}$ in $\mathrm{CH}_{3} \mathrm{OH}$. The IR spectrum of 2 showed the presence of hydroxyl (3498, 3458, $\left.3384 \mathrm{~cm}^{-1}\right)$, conjugated-ester carbonyl $\left(1714 \mathrm{~cm}^{-1}\right), \alpha, \beta$-unsaturated olefinic $\left(1645,1635 \mathrm{~cm}^{-1}\right)$ and aromatic ring $\left(1512,1456 \mathrm{~cm}^{-1}\right)$ functionalities. The ${ }^{1} \mathrm{H}-\mathrm{NMR}$ data of 2 (Table 2) demonstrated signals attributable to two pairs of 1,3,4-trisubstituted benzene ring signals at $\delta 7.04(1 \mathrm{H}, \mathrm{d}, J=8.25 \mathrm{~Hz}$, $\mathrm{H}-5), 6.85(1 \mathrm{H}, \mathrm{d}, J=1.75 \mathrm{~Hz}, \mathrm{H}-2), 6.61(1 \mathrm{H}, \mathrm{dd}, J=1.8,8.25 \mathrm{~Hz}, \mathrm{H}-6)$ and $6.90(1 \mathrm{H}, \mathrm{d}, J=1.4 \mathrm{~Hz}$, H-2'), $6.78\left(1 \mathrm{H}, \mathrm{m} \mathrm{H}-5^{\prime}\right), 6.77\left(1 \mathrm{H}, \mathrm{m}, \mathrm{H}-6^{\prime}\right)$; and a set of cinnamyl proton signals at $\delta 7.61(2 \mathrm{H}, \mathrm{m}, \mathrm{H}-2 "$, 6"), 7.43 (3H, m, H-3", 4", 5"), 7.68 (1H, d, $J=16 \mathrm{~Hz}, \mathrm{H}-7 "), 6.56$ (1H, d, $\left.J=16 \mathrm{~Hz}, \mathrm{H}-8^{\prime \prime}\right)$ in the aromatic region. In addition, resonances assignable to two aromatic methoxyl proton signals at $\delta 3.86$ $\left(3 \mathrm{H}, \mathrm{s}, 3^{\prime}-\mathrm{OCH}_{3}\right), 3.85\left(3 \mathrm{H}, \mathrm{s}, 3-\mathrm{OCH}_{3}\right)$, and an anomeric proton signal at $\delta 4.88\left(1 \mathrm{H}, \mathrm{d}, \mathrm{H}-1^{\prime \prime \prime}\right)$ were observed. Furthermore, in the ${ }^{1} \mathrm{H}-\mathrm{NMR}$ spectrum, an $O$-bearing methine proton signals at $\delta 4.72(1 \mathrm{H}, \mathrm{d}$, $\left.J=6.65 \mathrm{~Hz}, \mathrm{H}-7^{\prime}\right)$, an aliphatic methylene proton signals at $\delta 2.85(1 \mathrm{H}, \mathrm{dd}, J=4.7,13.5 \mathrm{~Hz}, \mathrm{H}-7 \mathrm{a}), 2.38$ $(1 \mathrm{H}, \mathrm{dd}, 11.55,13.3 \mathrm{~Hz}, \mathrm{H}-7 \mathrm{~b})$, two oxygenated methylene proton signals at $\delta 3.88(1 \mathrm{H}, \mathrm{dd}, J=6.5,8.35 \mathrm{~Hz}$, $\mathrm{H}-9 \mathrm{a}), 3.62(1 \mathrm{H}, \mathrm{m}, \mathrm{H}-9 \mathrm{~b}), 3.76\left(1 \mathrm{H}, \mathrm{m}, \mathrm{H}-9 \mathrm{a}^{\prime}\right), 3.60$ (1H, m, H-9 $\left.\mathrm{b}^{\prime}\right)$, and two aliphatic methine proton signals at $\delta 2.59(1 \mathrm{H}, \mathrm{m}, \mathrm{H}-8), 2.31\left(1 \mathrm{H}, \mathrm{m}, \mathrm{H}-8^{\prime}\right)$ were indicated. Besides the carbon resonances corresponding to the above units, the ${ }^{13} \mathrm{C}$-NMR spectrum (Table 2 ) and the DEPT indicated the presence of conjugated-ester carbonyl carbon signals at $\delta 168.37$ (C-9"); a set of a $\beta$-glucose moiety carbon signals at $\delta 102.87$ (C-1'"), 77.95 (C-3"'), 75.61 (C-5'"), 75.03 (C-2"'), 72.13 (C-4'"), 64.96 (C-6"'). The key HMBC correlations (Table 2 and Figure 3 ) from $3-\mathrm{OC}_{3} \underline{3}$ to $\mathrm{C}-3$; from 3'-OC$\underline{H}_{3}$ to C-3'; from H-7 to C-1, C-2, C-6, C-8 and C-9; from H-9 to C-7, C-7', C-8 and C-8'; from H-7' to C-1', C-2', C-6', C-8', C-9 and C-9'; from H-8' to C-1', C-7, C-7', C-8, C-9 and C-9'; and from H-9' to C-7, C-7', C-8 and C-8'; suggested that the aglycone should be lariciresinol [15]. Moreover, the HMBC correlations of H-1"'/C-4 confirmed that anomeric protons of $\beta$-glucose moiety was coupled with C-4. Meanwhile, the more deshielded proton of H-6"' ( $\delta_{\mathrm{H}} 4.55$ and 4.33) exhibited a HMBC correlation with C-9", which confirmed that the cinnamyl moiety was linked to C-6"'. In the NOESY spectrum of 2 (Figure 3), H-8' showed NOE correlations with $\mathrm{H}_{-2}{ }^{\prime}, \mathrm{H}-\mathrm{6}^{\prime}$ and $\mathrm{H}-8$, this experiment confirmed that $\mathrm{H}-8$ and $\mathrm{H}-\mathrm{8}^{\prime}$ are in cis-configuration, meanwhile, $\mathrm{H}-7^{\prime}$ and $\mathrm{H}-8^{\prime}$ are in trans-configuration. Comparison of the NMR data of (-)-lariciresinol 4'-(6"-O-feruloyl- $\beta$-D-glucopyranoside) [16] and compound 2 indicated they had very similar aglycone and sugar moieties, the only difference being the presence of a cinnamyl moiety instead of a feruloyl group. Compound $\mathbf{2}$ was hydrolysed by dilute acid, and the hydrolysate was identified to be D-glucose by the same way as described in the case of $\mathbf{1}$, Therefore the structure of $\mathbf{2}$ was determined to be (-)-lariciresinol 4-(6"'-O-cinnamyl- $\beta$-D-glucopyranoside).

Figure 3. Key HMBC and NOESY correlations in compound 2.
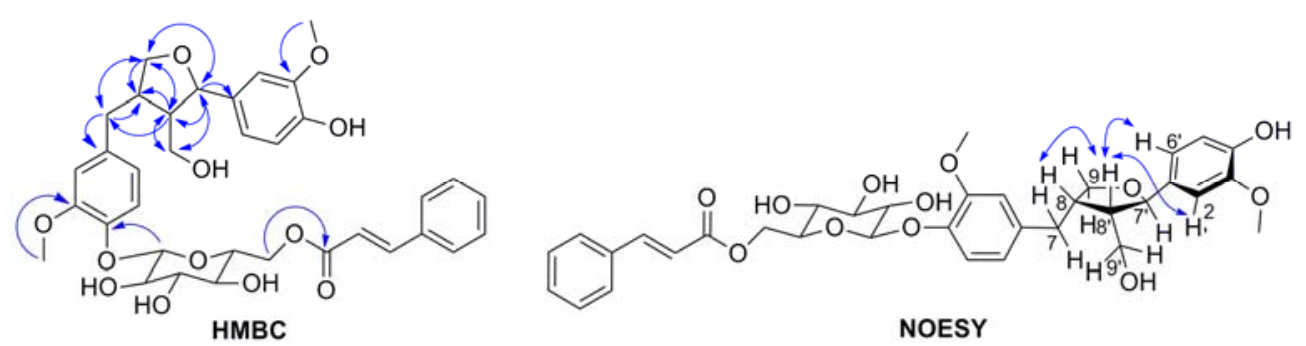
Table 2. ${ }^{1} \mathrm{H}-\mathrm{NMR}(500 \mathrm{MHz})$ and ${ }^{13} \mathrm{C}-\mathrm{NMR}(125 \mathrm{MHz})$ spectral data and $\mathrm{HMBC}$ correlations of compound 2 in $\mathrm{CD}_{3} \mathrm{OD}$.

\begin{tabular}{|c|c|c|c|}
\hline No. & $\delta_{\mathrm{C}}$ & $\delta_{\mathrm{H}}(J, \mathrm{~Hz})$ & HMBC \\
\hline 1 & 137.19 & & \\
\hline 2 & 114.46 & $6.85(1 \mathrm{H}, \mathrm{d}, 1.75)$ & $1,3,4,6,7$ \\
\hline 3 & 150.95 & & \\
\hline 4 & 146.21 & & \\
\hline 5 & 118.42 & $7.04(1 \mathrm{H}, \mathrm{d}, 8.25)$ & $1,2,3,4$ \\
\hline 6 & 122.34 & $6.61(1 \mathrm{H}, \mathrm{dd}, 1.8,8.25)$ & $2,3,4,5,7$ \\
\hline $7 a$ & \multirow{2}{*}{33.85} & $2.85(1 \mathrm{H}, \mathrm{dd}, 4.7,13.5)$ & $1,2,6,8,9$ \\
\hline $7 b$ & & $2.38(1 \mathrm{H}, \mathrm{dd}, 11.55,13.3)$ & $1,2,6,8$ \\
\hline 8 & 43.66 & $2.59(1 \mathrm{H}, \mathrm{m})$ & \\
\hline 9a & \multirow{2}{*}{73.53} & $3.88(1 \mathrm{H}, \mathrm{dd}, 6.5,8.35)$ & $7,7^{\prime}, 8^{\prime}$ \\
\hline $9 b$ & & $3.62(1 \mathrm{H}, \mathrm{m})$ & $7,7^{\prime}, 8,8^{\prime}$ \\
\hline $\mathbf{1}^{\prime}$ & 135.87 & & \\
\hline $2^{\prime}$ & 110.78 & $6.90(1 \mathrm{H}, \mathrm{d}, 1.4)$ & $1^{\prime}, 3^{\prime}, 4^{\prime}, 6^{\prime}, 7^{\prime}$ \\
\hline $3^{\prime}$ & 149.14 & & \\
\hline $4^{\prime}$ & 147.2 & & \\
\hline $5^{\prime}$ & 116.15 & $6.78(1 \mathrm{H}, \mathrm{m})$ & $1^{\prime}, 3^{\prime}, 4^{\prime}$ \\
\hline $6^{\prime}$ & 119.93 & $6.77(1 \mathrm{H}, \mathrm{m})$ & $2^{\prime}, 8^{\prime}$ \\
\hline $7^{\prime}$ & 84.11 & $4.72(1 \mathrm{H}, \mathrm{d}, 6.65)$ & $1^{\prime}, 2^{\prime}, 6^{\prime}, 8^{\prime}, 9,9^{\prime}$ \\
\hline $8^{\prime}$ & 54.15 & $2.31(1 \mathrm{H}, \mathrm{m})$ & $1^{\prime}, 7,7^{\prime}, 8,9,9^{\prime}$ \\
\hline $9 \mathbf{a}^{\prime}$ & \multirow{2}{*}{60.59} & $3.76(1 \mathrm{H}, \mathrm{m})$ & $7,7^{\prime}, 8,8^{\prime}$ \\
\hline $9 b^{\prime}$ & & $3.60(1 \mathrm{H}, \mathrm{m})$ & $7,7^{\prime}, 8,8^{\prime}$ \\
\hline 1" & 135.90 & & \\
\hline 2",6" & 129.47 & $7.61(2 \mathrm{H}, \mathrm{m})$ & 4", 7" \\
\hline 3",5" & 130.28 & $7.43(2 \mathrm{H}, \mathrm{m})$ & $1 "$ \\
\hline $4 "$ & 131.75 & $7.43(1 \mathrm{H}, \mathrm{m})$ & $1 ", 3 ", 5 "$ \\
\hline 7" & 146.59 & $7.68(1 \mathrm{H}, \mathrm{d}, 16)$ & 1", 2", 6", 8", 9" \\
\hline $8 "$ & 118.96 & $6.56(1 \mathrm{H}, \mathrm{d}, 16)$ & $1 ", 9 "$ \\
\hline 9" & 168.37 & & \\
\hline 1"' & 102.87 & $4.88(1 \mathrm{H}, \mathrm{d})$ & 4 \\
\hline $2^{\prime \prime \prime}$ & 75.03 & $3.53(1 \mathrm{H}, \mathrm{m})$ & 1"', 4"' \\
\hline 3"' & 77.95 & $3.52(1 \mathrm{H}, \mathrm{m})$ & 2"', 4"' \\
\hline 4"' & 72.13 & $3.43(1 \mathrm{H}, \mathrm{t})$ & 3"', 5"', 6"' \\
\hline $5 " '$ & 75.61 & $3.72(1 \mathrm{H}, \mathrm{m})$ & 1"', 4"', 6"' \\
\hline 6a"' & \multirow{2}{*}{64.96} & $4.55(1 \mathrm{H}, \mathrm{dd}, 2.2,11.75)$ & 5"', 9" \\
\hline $\mathbf{6 b}^{\prime \prime}$ & & $4.43(1 \mathrm{H}, \mathrm{dd}, 7.4,11.65)$ & 5"', 9" \\
\hline $3-\mathrm{OCH}_{3}$ & 56.85 & 3.85 & 3 \\
\hline 3'-OCH & 56.55 & 3.86 & $3^{\prime}$ \\
\hline
\end{tabular}

The known compounds 3-5 were identified by comparison of their spectroscopic data with literature values. HCT-116 and SW-620 cells were treated with three different concentrations of compounds for $24 \mathrm{~h}$. The antiproliferative effects of the five compounds on HCT-116 and SW-620 cells are shown in Table 3. None of the tested compounds had any significant effects on proliferation of HCT-116 cells and SW-620 cells at the tested concentrations of $1-10 \mu \mathrm{M}$. 
Table 3. Activity of compounds $\mathbf{1}-\mathbf{5}$ on proliferation of the HCT-116 and SW-620 cells.

\begin{tabular}{|c|c|c|c|c|c|c|}
\hline \multirow[b]{2}{*}{ Sample } & \multicolumn{3}{|c|}{ HCT-116 cells } & \multicolumn{3}{|c|}{ SW-620 cells } \\
\hline & $\begin{array}{c}10^{-4} \mathrm{~mol} / \mathrm{L} \\
\text { Inhibitory rate } \\
(\%) \\
\end{array}$ & $\begin{array}{c}10^{-5} \mathrm{~mol} / \mathrm{L} \\
\text { Inhibitory rate } \\
(\%) \\
\end{array}$ & $\begin{array}{c}10^{-6} \mathrm{~mol} / \mathrm{L} \\
\text { Inhibitory rate } \\
(\%) \\
\end{array}$ & $\begin{array}{c}10^{-4} \mathrm{~mol} / \mathrm{L} \\
\text { Inhibitory rate } \\
(\%) \\
\end{array}$ & $\begin{array}{c}10^{-5} \mathrm{~mol} / \mathrm{L} \\
\text { Inhibitory rate } \\
(\%) \\
\end{array}$ & $\begin{array}{c}10^{-6} \mathrm{~mol} / \mathrm{L} \\
\text { Inhibitory rate } \\
(\%) \\
\end{array}$ \\
\hline 1 & $5.383 \pm 2.187 * *$ & $16.150 \pm 0.504 * *$ & $13.329 \pm 0.367 * *$ & $6.967 \pm 0.262 * *$ & $5.026 \pm 0.713 * *$ & $1.218 \pm 1.016 * *$ \\
\hline 2 & $13.049 \pm 1.607 * *$ & $14.685 \pm 1.615 * *$ & $11.671 \pm 0.941 * *$ & $12.260 \pm 0.443 * *$ & $3.141 \pm 1.101 * *$ & $2.894 \pm 0.461 * *$ \\
\hline 3 & $12.086 \pm 1.404 * *$ & $13.157 \pm 0.748 * *$ & $5.943 \pm 0.931 * *$ & $24.119 \pm 1.128 * *$ & $8.605 \pm 0.745 * *$ & $5.863 \pm 0.287 * *$ \\
\hline 4 & $14.750 \pm 2.171 * *$ & $6.288 \pm 1.735 * *$ & $3.725 \pm 0.914 * *$ & $24.481 \pm 0.184 * *$ & $2.894 \pm 0.960 * *$ & $6.035 \pm 0.605 * *$ \\
\hline 5 & $19.079 \pm 0.440 * *$ & $13.868 \pm 0.373 * *$ & $2.972 \pm 0.734 * *$ & $26.499 \pm 0.453 * *$ & $3.769 \pm 0.799 * *$ & $2.646 \pm 1.577 * *$ \\
\hline Control & 0 & 0 & 0 & 0 & 0 & 0 \\
\hline
\end{tabular}

The data are expressed as mean $\pm \mathrm{SD}$ of three independent experiments. $(* * p<0.01 v s$. control).

\section{Experimental}

\subsection{General}

UV spectra were taken with a HALO DB-20R UV-VIS spectrophotometer. Optical rotations were recorded on a PerkinElmer Model 343 polarimeter. The IR spectra were recorded on a Shimadzu FTIR-8400S instrument. ESI-MS was performed on s Waters Quattro Premier instrument. The HR-ESIMS spectra was taken on an Agilent Technologies 6550 Q-TOF. 1D and 2D NMR spectra were recorded on a Bruker-AVANCE500 instrument with TMS as an internal standard. The analytical HPLC was performed on a Waters 2695 Separations Module coupled with a 2996 Photodiode Array Detector and a ODS-3 column $(4.6 \times 250 \mathrm{~mm}$, 5 ìm particles, Inertsill, Tokyo, Japan). Semipreparative HPLC was performed on a system comprising a Shimadzu LC-6AD pump equipped with a SPD-20A UV detector and a Ultimate XB-C18 $(10 \times 250 \mathrm{~mm}, 5 \mu \mathrm{m}$ particles $)$ or Allsphere ODS-2 $(10 \times 250 \mathrm{~mm}, 5 \mu \mathrm{m}$ particles). Sephadex LH-20 was purchased from GE Healthcare Bio-Sciences AB (Uppsala, Sweden). MCI GEL was from Mitsubishi Chemical Corporation (Tokyo, Japan). C-18 (40-75 $\mu \mathrm{m})$ silicagel was purchased from SiliCycle Corporation (Quebec, Canada). D101 was from Sunresin New Materials Co. Ltd. (Xi'an, China). Silica gel was purchased from Qingdao Haiyang Chemical Group Corporation (Qingdao, China).

\subsection{Plant Materials}

The root of Jasminum giraldii Diels. was collected on October in 2009 in Shanxi, China, and authenticated by Prof. Yaowu Guo. A voucher specimen has been deposited in the Department of Collaborative Innovation Center for Chinese Medicine in Qingba Mountains, School of Pharmacy the Fourth Military Medical University.

\subsection{Extraction and Isolation}

The root of $J$. giraldii $(20 \mathrm{~kg})$ was powdered and extracted with EtOH $(100 \mathrm{~L}, 3 \mathrm{~h} \times 4)$ under reflux. Evaporation of the solvent under reduced pressure gave the EtOH extract. The combined extract was applied to D101 and eluted with EtOH- $\mathrm{H}_{2} \mathrm{O}$ to obtain the $95 \% \mathrm{EtOH}$ eluate $(15 \mathrm{~L})$. The eluate was 
evaporated under vacuum and freeze dried to yield a powder $(895 \mathrm{~g})$, which was suspended in $\mathrm{H}_{2} \mathrm{O}$ and partitioned successively with petroleum ether, EtOAc and $n-\mathrm{BuOH}$. The EtOAc fraction was subjected to silica-gel column chromatography eluting with $\mathrm{CHCl}_{3}-\mathrm{MeOH}(100: 0$ to $1: 1)$ to obtain 33 fractions (Fr.1-Fr.33). Fr.28 was further separated by flash chromatography over MCI, eluting with a gradient of $\mathrm{MeOH}(0-100 \%)$ in $\mathrm{H}_{2} \mathrm{O}$ to obtain seven fractions (Fr.28-1-Fr.28-7). Repeated chromatography of Fr.28-1 with silica gel $\left(\mathrm{MeOH} / \mathrm{CHCl}_{3}, 30: 1-5: 1\right)$, Sephadex LH-20 $\left(\mathrm{CHCl}_{3} / \mathrm{MeOH}, 1: 1\right)$, prep-TLC and prep-HPLC gave compounds 1 ( $8 \mathrm{mg}), 2(26 \mathrm{mg}), 4(28 \mathrm{mg}), \mathrm{Fr} .21$ was subjected to silica gel (200-300 mesh) column chromatography and eluted with $\mathrm{CHCl}_{3} / \mathrm{MeOH}(100: 0-50: 1)$ to obtain five fractions (Fr.21-1-Fr.21-5). Fr.21-5 was further separated repeated with silica gel column (petroleum ether/acetone, 8:1-5:1), Sephadex LH-20 $\left(\mathrm{CHCl}_{3} / \mathrm{MeOH}, 1: 1\right)$, prep-TLC and prep-HPLC to afford compounds 3 (120 $\mathrm{mg}$ ) and $\mathbf{5}(5 \mathrm{mg})$.

\subsection{Spectral Data}

Ethylconiferin (1): white powder; $\mathrm{C}_{18} \mathrm{H}_{26} \mathrm{O}_{8} ;[\alpha]_{D}^{20}$ : $-19.76\left(\mathrm{c} 0.1, \mathrm{CH}_{3} \mathrm{OH}\right) ; \mathrm{UV}_{\max }(\mathrm{MeOH}) \lambda_{\max } 290$, 259 and $213 \mathrm{~nm}$; IR (KBr) $v_{\max }$ 3496, 3444, 3367, 2979, 2939, 2867, 1649, 1510, 1454, 1128, 1089, 1047, $1027 \mathrm{~cm}^{-1}$; HR-TOF-MS $m / z 393.1525[\mathrm{M}+\mathrm{Na}]^{+}$(calcd for $\mathrm{C}_{18} \mathrm{H}_{26} \mathrm{NaO}_{8} ; 393.1525$ ); ${ }^{1} \mathrm{H}-\mathrm{NMR}$ $\left(500 \mathrm{MHz}, \mathrm{CD}_{3} \mathrm{OD}\right)$ and ${ }^{13} \mathrm{C}-\mathrm{NMR}\left(125 \mathrm{MHz}, \mathrm{CD}_{3} \mathrm{OD}\right)$ data, see Table 1.

(-)-Lariciresinol 4-(6"'-O-cinnamyl- $\beta$-D-glucopyranoside) (2): white powder; $\mathrm{C}_{35} \mathrm{H}_{40} \mathrm{O}_{12} ;[\alpha]_{D}^{20}$ : -10.91 (c 0.1, MeOH); UV $\mathrm{Uax}_{\max }(\mathrm{MeOH}) \lambda_{\max } 278,216$ and $203 \mathrm{~nm}$; IR(KBr) $v_{\max }$ 3498, 3458, 3384, 2925, 2854, $1714,1645,1635,1512,1456,1269,1226,1161,1124,1105,1072,1035 \mathrm{~cm}^{-1}$; HR-TOF-MS $\mathrm{m} / z$ $653.2590[\mathrm{M}+\mathrm{H}]^{+}$(calcd for $\left.\mathrm{C}_{35} \mathrm{H}_{41} \mathrm{O}_{8} ; 653.2590\right) ;{ }^{1} \mathrm{H}-\mathrm{NMR}\left(500 \mathrm{MHz}, \mathrm{CD}_{3} \mathrm{OD}\right)$ and ${ }^{13} \mathrm{C}-\mathrm{NMR}(125 \mathrm{MHz}$, $\left.\mathrm{CD}_{3} \mathrm{OD}\right)$ data, see Table 2.

(+)-Pinoresinol (3): yellow powder; $\mathrm{C}_{20} \mathrm{H}_{22} \mathrm{O}_{6} ;[\alpha]_{D}^{20}:+46.0$ (c 0.1, MeOH); $\mathrm{UV}_{\max }(\mathrm{MeOH}) \lambda_{\max } 280$, 231 and $207 \mathrm{~nm}$; IR (KBr) $v_{\max }$ 3504, 3438, 3379, 2958, 2939, 2860, 1604, 1514, 1460, 1438, 1271, $1029 \mathrm{~cm}^{-1}$; ESI-MS m/z 357 [M-H] $]^{-}{ }^{1} \mathrm{H}-\mathrm{NMR}\left(500 \mathrm{MHz}, \mathrm{Me}_{2} \mathrm{CO}-d_{6}\right) \delta 7.47$ (2H, s, 4-OH and 4'-OH), $6.99\left(2 \mathrm{H}, \mathrm{d}, J=1.7 \mathrm{~Hz}, \mathrm{H}-2,2^{\prime}\right), 6.84\left(2 \mathrm{H}, \mathrm{dd}, J=1.7,8.1 \mathrm{~Hz}, \mathrm{H}-6,66^{\prime}\right), 6.79$ (2H, d, $J=8.1 \mathrm{~Hz}, \mathrm{H}-5$, $\left.5^{\prime}\right), 4.67\left(2 \mathrm{H}, \mathrm{d}, J=4.15 \mathrm{~Hz}, \mathrm{H}-7,7^{\prime}\right), 4.20$ (2H, dd, $J=6.95,8.95 \mathrm{~Hz}, \mathrm{H}-9 \mathrm{a}, 9$ 'a), 3.84(6H, s, 3-OCH ${ }_{3}$ and 3'- $\left.\mathrm{OCH}_{3}\right), 3.81\left(2 \mathrm{H}, \mathrm{dd}, J=3.7,9.1 \mathrm{~Hz}, \mathrm{H}-9 \mathrm{~b}, 9 \mathrm{\prime}\right.$ b), $3.08\left(2 \mathrm{H}, \mathrm{m}, \mathrm{H}-8,88^{\prime}\right)$; ${ }^{13} \mathrm{C}-\mathrm{NMR}(125 \mathrm{MHz}$, $\left.\mathrm{Me}_{2} \mathrm{CO}-d_{6}\right) \delta 148.40\left(\mathrm{C}-3,3^{\prime}\right), 146.94\left(\mathrm{C}-4,4^{\prime}\right), 134.27$ (C-1, 1'), 119.69 (C-6, 6'), 115.62 (C-5, 5'), $110.67\left(\mathrm{C}-2,2^{\prime}\right), 86.72\left(\mathrm{C}-7,7^{\prime}\right), 72.29\left(\mathrm{C}-9,9^{\prime}\right), 56.33\left(3-\mathrm{OCH}_{3}\right.$ and $\left.3^{\prime}-\mathrm{OCH}_{3}\right), 55.32\left(\mathrm{C}-8,8^{\prime}\right)$.

(+)-Cycloolivil (4): yellow powder; $\mathrm{C}_{20} \mathrm{H}_{24} \mathrm{O}_{7} ;[\alpha]_{D}^{20}$ : +43.2 (c 0.1, $\mathrm{MeOH}$ ); $\mathrm{UV}_{\max }(\mathrm{MeOH}) \lambda_{\max } 283,230$ and $204 \mathrm{~nm}$; IR (KBr) $v_{\max } 3517,3444,3313,3201,2939,2900,2835,1699,1598,1517,1465,1446$, 1433, 1373, 1269, 1236, 1215, 1149, 1124, 1022, 881, 858, 838, $811 \mathrm{~cm}^{-1}$; ESI-MS m/z $375[\mathrm{M}-\mathrm{H}]^{-}$; ${ }^{1} \mathrm{H}-\mathrm{NMR}\left(500 \mathrm{MHz}, \mathrm{CD}_{3} \mathrm{OD}\right) \delta 6.76(1 \mathrm{H}, \mathrm{d}, J=8.0 \mathrm{~Hz}, \mathrm{H}-5), 6.76(1 \mathrm{H}, \mathrm{d}, J=1.6 \mathrm{~Hz}, \mathrm{H}-2), 6.67(1 \mathrm{H}$, dd, $J=1.7,7.95$ Hz, H-6), 6.63 (1H, s, H-5'), 6.18 (1H, s, H-2'), 4.03 (1H, d, J=11.65 Hz, H-7), 3.80 $\left(3 \mathrm{H}, \mathrm{s}, \mathrm{OCH}_{3}\right), 3.77$ (2H, m, H-9a, 9'a), $3.74\left(3 \mathrm{H}, \mathrm{OCH}_{3}\right), 3.59\left(2 \mathrm{H}, \mathrm{m}, \mathrm{H}-9 \mathrm{~b}, 9{ }^{\prime} \mathrm{b}\right), 3.23$ (1H, d, J= 16.65 Hz, H-7'a), 2.62 (1H, d, $J=16.65 \mathrm{~Hz}, \mathrm{H}-7 \mathrm{\prime}$ ) $), 2.04$ (1H, m, H-8); ${ }^{13} \mathrm{C}-\mathrm{NMR}\left(125 \mathrm{MHz}, \mathrm{CD}_{3} \mathrm{OD}\right) \delta$ 149.27 (C-3), 147.64 (C-3'), 146.25 (C-4), 145.44 (C-4'), 138.62 (C-1'), 133.69 (C-1), 126.57 (C-6'), 
123.71 (C-6), 117.48 (C-5), 116.15 (C-5'), 114.06 (C-2), 113.09 (C-2'), 75.10 (C-8'), 69.55 (C-9), 60.98 $\left(\mathrm{C}-9\right.$ '), $56.52\left(\mathrm{OCH}_{3}\right), 56.49\left(\mathrm{OCH}_{3}\right), 47.69(\mathrm{C}-8), 45.03(\mathrm{C}-7), 40.06\left(\mathrm{C}-7^{\prime}\right)$.

Nobiletin (5): white powder; $\mathrm{C}_{9} \mathrm{H}_{12} \mathrm{O}_{2} ;[\alpha]_{D}^{20}:+3.69$ (c 0.1, MeOH); $\mathrm{UV}_{\max }(\mathrm{MeOH}) \lambda_{\max } 328,269,249$ and $208 \mathrm{~nm}$. IR (KBr) $v_{\max } 2947,2854,1629,1598,1514,1461,1377,1278,1149,1107,1078,1012$, 973, $838 \mathrm{~cm}^{-1}$; ESI-MS m/z $403[\mathrm{M}+\mathrm{H}]^{+}$; ${ }^{1} \mathrm{H}-\mathrm{NMR}\left(500 \mathrm{MHz}, \mathrm{Me}_{2} \mathrm{CO}-d_{6}\right) \delta 7.66(1 \mathrm{H}, \mathrm{dd}$, $J=2.15,8.5 \mathrm{~Hz}, \mathrm{H}-6$ ') 7.59 (1H, d, $\left.J=2.05 \mathrm{~Hz}, \mathrm{H}-2^{\prime}\right), 7.15$ (1H, d, $J=8.5 \mathrm{~Hz}, \mathrm{H}-5$ '), 6.62 (1H, s, H-3), $4.07\left(3 \mathrm{H}, \mathrm{s}, \mathrm{OCH}_{3}\right), 4.04\left(3 \mathrm{H}, \mathrm{s}, \mathrm{OCH}_{3}\right), 3.96\left(3 \mathrm{H}, \mathrm{s}, \mathrm{OCH}_{3}\right), 3.92\left(3 \mathrm{H}, \mathrm{s}, \mathrm{OCH}_{3}\right), 3.89\left(3 \mathrm{H}, \mathrm{s}, \mathrm{OCH}_{3}\right)$, $3.85\left(3 \mathrm{H}, \mathrm{s}, \mathrm{OCH}_{3}\right) ;{ }^{13} \mathrm{C}-\mathrm{NMR}\left(125 \mathrm{MHz}, \mathrm{Me}_{2} \mathrm{CO}-d_{6}\right) \delta 176.74(\mathrm{C}-4), 161.67(\mathrm{C}-2), 153.38(\mathrm{C}-4$ '), 152.4 (C-7), 150.72 (C-3'), 149.22 (C-9), 148.64 (C-8), 145.15 (C-5), 139.24 (C-6), 124.85 (C-1'), 120.39 (C-6'), 115.91 (C-10), 112.69 (C-5'), 110.01 (C-2'), 107.29 (C-3), 62.44, 62.37, 62.04, 61.98, 56.34, $56.31\left(5-\mathrm{OCH}_{3} 6-\mathrm{OCH}_{3}, 7-\mathrm{OCH}_{3}, 8-\mathrm{OCH}_{3}, 3^{\prime}-\mathrm{OCH}_{3}\right.$ and $\left.4 '-\mathrm{OCH}_{3},\right)$.

\subsection{Enzymatic Hydrolysis of $\mathbf{1}$}

A solution of compound 1 in $\mathrm{H}_{2} \mathrm{O}(3 \mathrm{~mL})$ was hydrolysed with $\beta$-glucosidase (10 $\mathrm{mg}$, Almonds Lot 1264252, Sigma-Aldrich, St. Louis, MO, USA) at $37{ }^{\circ} \mathrm{C}$ for 24 or $36 \mathrm{~h}$. The reaction mixture was extracted with EtOAc for three times $(3 \times 3 \mathrm{~mL})$ to yield the EtOAc exrract and $\mathrm{H}_{2} \mathrm{O}$ phase after removing the solvents. The aqueous phase of the hydrolysate of compound 1 was subjected to $\mathrm{CC}$ over silica gel eluted with $\mathrm{MeCN} / \mathrm{H}_{2} \mathrm{O}(8: 1)$ to yield glucose with positive optical rotations, and the value of $[\alpha]_{D}^{20}$ : was +47.2 (c $\left.0.16, \mathrm{H}_{2} \mathrm{O}\right)$. The solvent system $\mathrm{MeCN} / \mathrm{H}_{2} \mathrm{O}(6: 1)$ was used for the TLC analysis of glucose and authentic sugar samples.

\subsection{Acid Hydrolysis of 2}

A solution of compound 2 in $0.5 \mathrm{M} \mathrm{H}_{2} \mathrm{SO}_{4}(2.0 \mathrm{~mL})$ was heated under reflux for $3 \mathrm{~h}$. After cooling, the reaction mixture was diluted with $\mathrm{H}_{2} \mathrm{O}$, neutralized with $\mathrm{BaCO}_{3}$, then filtered. The solution was extracted with EtOAc for three times $(3 \times 3 \mathrm{~mL})$ to obtained the EtOAc extract and $\mathrm{H}_{2} \mathrm{O}$ layer after removing the solvents. The aqueous layer of the hydrolysate of compound $\mathbf{2}$ was evaporated and to yield glucose with positive optical rotations, and the value of $[\alpha]_{D}^{20}$ : was $+42.5\left(c 0.11, \mathrm{H}_{2} \mathrm{O}\right)$. The solvent system $\mathrm{MeCN} / \mathrm{H}_{2} \mathrm{O}(6: 1)$ was used for the TLC analysis of glucose and authentic sugar samples.

\subsection{Bioassay for Cytotoxic Activity}

The human colon cell line HCT-116 and SW620 was obtained from the Shanghai Institute of Cell Biology. The cells were maintained in DMEM medium supplemented with $10 \% \mathrm{FBS}$, penicillin $(100 \mathrm{U} / \mathrm{mL})$ and streptomycin $(100 \mathrm{mg} / \mathrm{mL})$ at $37{ }^{\circ} \mathrm{C}$ in a humidified atmosphere with $5 \% \mathrm{CO}_{2}$. Cell proliferation of HCT-116 cells was assessed by conducting colorimetric MTT cell proliferation assay. Briefly, a limited number of growing cells were seeded into 96-well cell culture plate and were maintained for $24 \mathrm{~h}$ so that they became attached to the bottom of the well. The medium was aspirated and new medium $(200 \mu \mathrm{L})$ including three groups of concentrations $\left(10^{-4}, 10^{-5}, 10^{-6} \mathrm{M}\right)$ of compounds were added to parallel wells. The cells were incubated at $37^{\circ} \mathrm{C}$ in a humidified atmosphere for $24 \mathrm{~h}$. Twenty $\mu \mathrm{L}$ of MTT solution was added and the incubation continued for $4 \mathrm{~h}$. The pure formazan product was then solubilized by $150 \mu \mathrm{L}$ DMSO. The plates were read at $570 \mathrm{~nm}$ using a microtiter plate reader. 


\section{Conclusions}

Compounds 1, 2 were new glycosides and together with compounds 3-5 were isolated from the Jasminum genus for the first time. Compounds 1-5 were assayed for their cytotoxic activity, and the data proved that none of the compounds had any significant inhibitory activities on proliferation of HCT-116 cells and SW-620 cells at the concentrations of $1-10 \mu \mathrm{M}$.

\section{Supplementary Materials}

Supplementary materials can be accessed at: http://www.mdpi.com/1420-3049/18/4/4766/s1.

\section{Acknowledgments}

Financial support from the National Natural Sciences Foundation of China (NNSFC; Grant No. 81072671), and the National Program on Key Basic Research Project of China (973 Program; Grant No. 2010CB535002) is acknowledged.

\section{References}

1. Zhang, M.Z.; Qiu, L.Q. Flora of China; Science Press: Beijing, China, 1992; Volume 61, p. 183.

2. Huong, N.T.; Cu, N.K.; Quy, T.V.; Zidorn, C.; Ganzera, M.; Stuppner, H. A new phenylpropanoid glycoside from Jasminum subtriplinerve Blume. J. Asian Nat. Prod. Res. 2008, 10, 1035-1038.

3. Shen, Y.C.; Lin, S.L. New secoiridoid glucosides from Jasminum lanceolarium. Planta Med. 1996, $62,515-518$.

4. Tanahashi, T.; Shimada, A.; Nagakura, N.; Nayeshiro, H. Jasamplexosides A, B and C: Novel dimeric and trimeric secoiridoid glucosides from Jasminum amplexicaule. Planta Med. 1992, 58, $552-555$.

5. Somanadhan, B.; Smitt, U.W.; George, V.; Pushpangadan, P.; Rajasekharan, S.; Duus, J.O.; Nyman, U.; Olsen, C.E.; Jaroszewski, J.W. Angiotensin converting enzyme (ACE) inhibitors from Jasminum azoricum and Jasminum grandiflorum. Planta Med. 1998, 64, 246-250.

6. Huan, M.; Cui, H.; Teng, Z.; Zhang, B.; Wang, J.; Liu, X.; Xia, H.; Zhou, S.; Mei, Q. In vivo anti-tumor activity of a new doxorubicin conjugate via alpha-linolenic acid. Biosci. Biotechnol. Biochem. 2012, 76, 1577-1579.

7. Wang, Z.; Zhu, S.; Shen, M.; Liu, J.; Wang, M.; Li, C.; Wang, Y.; Deng, A.; Mei, Q. STAT3 is involved in esophageal carcinogenesis through regulation of Oct-1. Carcinogenesis 2012, 34, 678-688.

8. Zhang, D.; Sun, Y.; Yue, Z.; Li, Q.; Meng, J.; Liu, J.; Hekong, X.; Jiang, F.; Mi, M.; Liu, L.; et al. Apple polysaccharides induce apoptosis in colorectal cancer cells. Int. J. Mol. Med. 2012, 30, 100-106.

9. Zhao, H.; Yang, Z.; Wang, X.; Zhang, X.; Wang, M.; Wang, Y.; Mei, Q.; Wang, Z. Triptolide inhibits ovarian cancer cell invasion by repression of matrix metalloproteinase 7 and 19 and upregulation of E-cadherin. Exp. Mol. Med. 2012, 44, 633-641. 
10. Cowan, S.; Stewart, M.; Abbiw, D.K.; Latif, Z.; Sarker, S.D.; Nash, R.J. Lignans from Strophanthus gratus. Fitoterapia 2001, 72, 80-82.

11. Ghogomu-Tih, R.; Bodo, B.; Nyasse, B.; Sondengam, B.L. Isolation and identification of (-)-Olivil and (+)-Cycloolivil from Stereospermum kunthianum. Planta Med. 1985, 51, 464.

12. Hamdan, D.; El-Readi, M.Z.; Tahrani, A.; Herrmann, F.; Kaufmann, D.; Farrag, N.; El-Shazly, A.; Wink, M. Chemical composition and biological activity of Citrus jambhiri Lush. Food Chem. 2011, 127, 394-403.

13. Hudson, C.S.; Dale, J.K. Studies on the forms of D-glucose and their mutarotation. J. Am. Chem. Soc. 1917, 39, 320-328.

14. Ping, H.; Gloria, K.; Pepter, G. Phenylpropanoid glycosides from Typhonium flagelliforme (Araceace). Nat. Prod. Res. Dev. 2004, 16, 403-405.

15. Duh, C.Y.; Phoebe, C.H., Jr.; Pezzuto, J.M.; Kinghorn, A.D.; Farnsworth, N.R. Plant anticancer agents, XLII. Cytotoxic constituents from Wikstroemia elliptica. J. Nat. Prod. 1986, 49, 706-709.

16. Ouyang, M.-A.; Wein, Y.-S.; Kuo, Y.-H. Four new lariciresinol-based lignan glycosides from the roots of rhus javanica var. roxburghiana. Helv. Chim. Acta 2007, 90, 1099-1106.

Sample Availability: Samples of the compounds 2-4 are available from the authors.

(C) 2013 by the authors; licensee MDPI, Basel, Switzerland. This article is an open access article distributed under the terms and conditions of the Creative Commons Attribution license (http://creativecommons.org/licenses/by/3.0/). 